\title{
Recent Phenomenological Predictions for Central Exclusive Production at the LHC
}

\author{
B. E. Cox, F. K. Loebinger and A. D. Pilkington * \\ Department of Physics and Astronomy, The University of Manchester, \\ Manchester M13 9PL, UK.
}

\begin{abstract}
We present the latest luminosity dependent background predictions for central exclusive processes at the LHC. The effect of these predictions on the potential observation of a Higgs boson in the MSSM is also discussed.
\end{abstract}

\section{Introduction}

The central exclusive process (CEP) is defined as $p p \rightarrow p+X+p$ and no other hadronic activity [2]. During the interaction, the protons remain intact, are deflected through very small angles and typically lose less than $1 \%$ of their energy. The mass of the central system can be reconstructed from just the outgoing proton momentum. Furthermore, to a very good approximation the central system, $X$, is produced in a $0^{++}$state. Thus, by tagging the outgoing protons and measuring the momenta, the mass and quantum numbers of a resonance is known regardless of the decay products.

In this contribution, we give a brief insight into the luminosity dependent backgrounds to CEP, which have only recently been evaluated. We assume that forward proton detectors have been installed $420 \mathrm{~m}$ from the interaction point at ATLAS (and CMS) as detailed by the FP420 proposal [3].

\section{Luminosity dependent backgrounds}

The luminosity dependent (or overlap) backgrounds occur due to the large number of interactions in each bunch crossing at the LHC. The largest contribution, which we denote as $[\mathrm{p}][\mathrm{X}][\mathrm{p}]$, comes from a three-fold coincidence between an inclusive hard scatter event, $[\mathrm{X}]$, and two single diffractive events, $[\mathrm{p}]$, each of which produces a proton within the acceptance of the forward detectors. The luminosity dependence of this background arises because the probability of single diffractive events occurring in a specific bunch crossing increases with the number of interactions in the bunch crossing.

The cross section, $\sigma$, can be estimated by

$$
\sigma=\sigma_{[X]} \sum_{N=3}^{\infty} \frac{\lambda^{N} e^{-\lambda}}{N !} P_{2[p]}(N-1)
$$

where $\sigma_{[X]}$ is the cross section of the inclusive hard scatter event and $\lambda$ is the average number of interactions in a bunch crossing at the LHC. The $P_{2[p]}(n)$ factor is the probability that, given $n$ interactions, there are at least two single diffractive events, each of which produces a proton within the acceptance of a forward detector. This probability is calculated using a trinomial distribution which utilises the fraction, $f_{s d}$, of events at the LHC that are single diffractive and produce an outgoing proton within the acceptance of a forward detector.

*This work was funded in the UK by PPARC/STFC. 


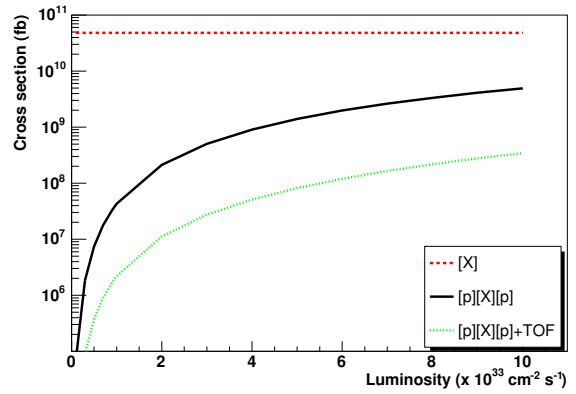

(a)

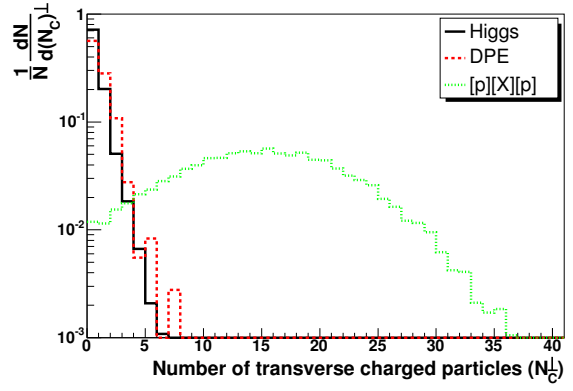

(b)

Figure 1: The cross section for the overlap di-jet cross section $\left(E_{T}>40 \mathrm{GeV}\right)$ is shown in (a) as a function of luminosity. Figure (b) shows the number of charged particles that are perpendicular in azimuth to the leading jet.

There is a consensus between theoretical predictions and Monte Carlo event generators that $f_{s d} \sim 0.01$ for $0.002 \leq \xi \leq 0.02$, which is the approximate fractional momentum loss acceptance of FP420. Figure 1(a) shows the cross section (fb) for overlap di-jet events at the LHC after requiring that the parton has a transverse momentum of $40 \mathrm{GeV}$. The cross section dependence is approximately quadratic. The luminosity dependent background can also be reduced by using proton time-of-flight (TOF) information to construct an 'event vertex', which can be matched to the di-jet vertex (see [4]).

The overlap background is reduced further by the clean nature of the central exclusive events. Di-jets from the inclusive event will be colour connected to the proton remants and the occurance of multi-parton interactions means that there will be so-called underlying event. In figure 1(b), we compare the number of charged particles that are perpendicular in azimuth to the leading jet. The signal events are generated by the ExHuME MC [5] and the inclusive di-jet events by HERWIG [6] with JIMMY [7] used for the underlying event. To create the overlap background, the protons are added in on an event by event basis with a distribution in $\xi$ and $t$ given by [8]. By requiring that there are few charged particles in this region, the overlap background can be additionally reduced by a factor of $\sim 100$. This however, is dependent on the MC tune used to generate the inclusive events.

\section{Implications for MSSM Higgs boson observation}

To illustrate the effect of this luminosity dependent background, we examine the potential observation of a light MSSM Higgs boson in the $b \bar{b}$ decay channel. Within the $m_{h}^{\text {max }}$ scenario, with $\tan \beta=40, m_{A}=120 \mathrm{GeV}$ and $\mu=200 \mathrm{GeV}$, the cross section of $h \rightarrow b \bar{b}$ is $17.9 \mathrm{fb}$ and the Higgs boson has a mass of $119.5 \mathrm{GeV}$ with a width of $3.2 \mathrm{GeV}$. The relevant backgrounds to this process are CEP $b \bar{b}$ and $g g$ production, $b \bar{b}$ production via double pomeron exchange (DPE) and overlap. A transverse energy cut of $45 \mathrm{GeV}$ is imposed on the leading jet in order to reduce the QCD backgrounds. The non-CEP backgrounds are reduced by comparing the 


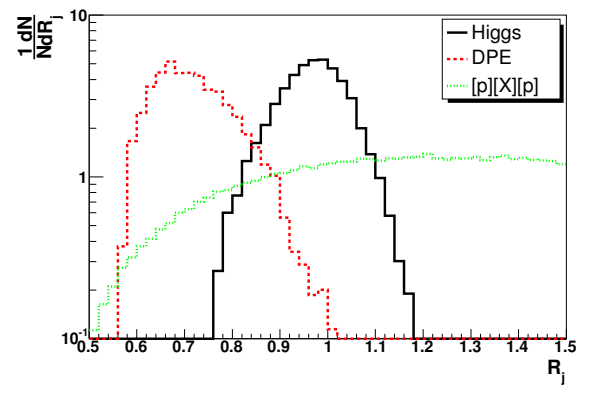

(a)

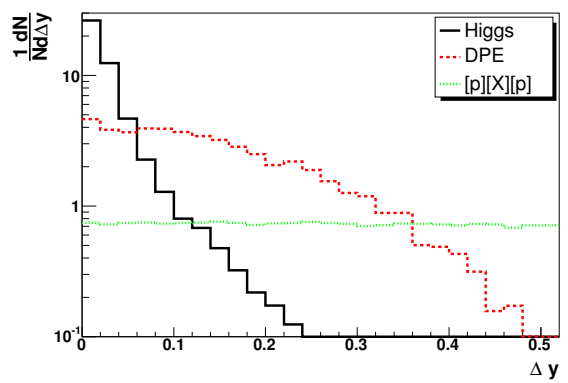

(b)

Figure 2: The $R_{j}$ distribution for signal, DPE and overlap backgrounds (a). The corresponding $\Delta y$ distributions are shown in (b).

kinematic information from FP420 to the di-jet system.

Firstly, we use the di-jet mass fraction, which compares the mass of the di-jet system to the central mass measured by FP420. We use the $R_{j}$ definition [9] for the di-jet mass fraction as it is less affected by final state radiation effects. For central exclusive backgounds, we expect that $R_{j} \sim 1$. Figure 2 (a) compares the $R_{j}$ distributions for the signal and background events after smearing the particles with the intrinsic resolution of the ATLAS detector. The DPE backgrounds, generated with the POMWIG MC [10], have a smaller $R_{j}$ than CEP processes due to the presence of pomeron remnants. The overlap backgrounds typically have a large range of $R_{j}$ values because the protons do not originate from the same interaction as the di-jets and the proton kinematics, in general, do not match the di-jet system. Figure 2(b) shows the equivalent plots for the $\Delta y$ variable, which is the modulus of the difference between the rapidity of the central system as measured by FP420 and the average rapidity of the two jets. The CEP events are peaked at zero as expected and the backgrounds spread over a large range in $\Delta y$. An exclusive enriched sample can be obtained by requiring that $0.75 \leq R_{j} \leq 1.1$ and $\Delta y \leq 0.06$. After these cuts, the DPE backgrounds are negligible.

The signal cross section is $0.6 \mathrm{fb}$ before trigger efficiency. The largest loss in signal is from FP420 acceptance $(28 \%)$, double b-tagging efficiency $(36 \%)$ and the jet $E_{T}$ requirement $(50 \%)$. The CEP backgrounds are reduced to $2.3 \mathrm{fb}$ and the overlap background to 0.04 (5.5) fb at low (high) luminosity. However, these backgrounds are spread over the mass range $80-160 \mathrm{GeV}$, whereas the signal is smeared only by the FP420 mass resolution of approximately $2 \mathrm{GeV}$. To estimate the significance of a potential observation, events are selected at random for each process to create a 'data sample'. The resulting mass distribution is fitted with a null hypothesis and a background plus gaussian signal hypothesis. The significance is estimated from the $\Delta \chi^{2}$ of the two fits. The process is repeated for many 'samples' and an averaged significance obtained.

The limiting factor is the trigger strategy as shown in figure 3 . The signal from the forward detectors arrive too late to be included in the level one trigger and the events must therefore be retained by information from the central detector. The forward proton 


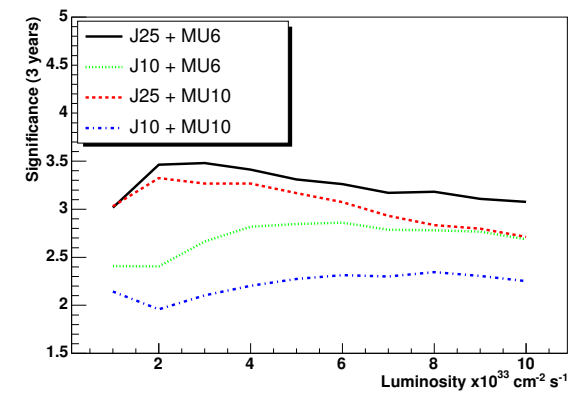

(a)

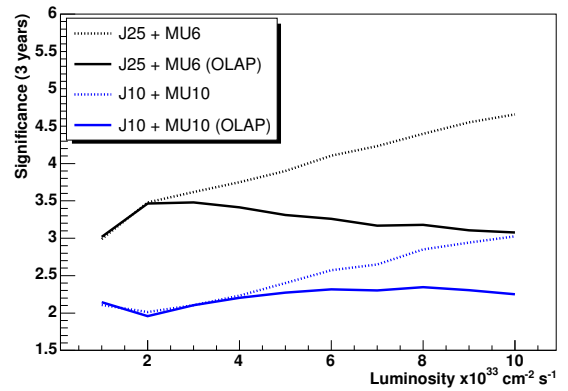

(b)

Figure 3: The significance of observation as a function of luminosity (a). The different curves label different trigger strategies, i.e. J10 is a jet-rate trigger which is pre-scaled to 10 $\mathrm{kHz}$ and MU6 is a $6 \mathrm{GeV}$ muon trigger. Fig (b) shows the effect of the overlap background.

information could then be used at level 2 to substantially reduce the rate for non-diffractive events. We define two complementary trigger strategies. The first is a low transverse momentum muon in conjunction with a jet with $E_{T}>40 \mathrm{GeV}$. The second is to have a di-jet trigger with $E_{T}>40 \mathrm{GeV}$, which is pre-scaled to a given rate. Figure 3(a) shows the significance after three years of for a combination of these triggers.

Figure 3(b) shows the effect of the overlap background at high luminosity. Without overlap, the significance can approach 5 in the best case scenario and one would expect a significance of $\sim 3$ for the more conservative triggers. With overlap, the significance is restricted to $\sim 3$ in the best case scenario. It may be possible to increase the rejection of the overlap background with improved proton time-of-flight measurements. Furthermore, the analysis presented here has not utilised forward detectors at $220 \mathrm{~m}$. The significance will increase when the analyses are combined.

\section{References}

[1] Slides: http://indico. cern. ch/contributionDisplay . py? contribId=68\&sessionId=7\&conf Id=9499

[2] A. De Roeck, V. A. Khoze, A. D. Martin, R. Orava and M. G. Ryskin, Eur. Phys. J. C 25 (2002) 391 [arXiv:hep-ph/0207042].

J. R. Forshaw, arXiv:hep-ph/0508274.

[3] M. G. Albrow et al., CERN-LHCC-2005-025.

[4] A. D. Pilkington, Status of the FP420 project, These proceedings.

[5] J. Monk and A. Pilkington, Comput. Phys. Commun. 175 (2006) 232 [arXiv:hep-ph/0502077].

[6] G. Corcella et al., arXiv:hep-ph/0210213.

[7] J. M. Butterworth, J. R. Forshaw and M. H. Seymour, Z. Phys. C 72 (1996) 637 [arXiv:hep-ph/9601371].

[8] V. A. Khoze, A. D. Martin and M. G. Ryskin, Phys. Lett. B 643 (2006) 93 [arXiv:hep-ph/0609312].

[9] V. A. Khoze, A. D. Martin and M. G. Ryskin, Eur. Phys. J. C 48 (2006) 467 [arXiv:hep-ph/0605113].

[10] B. E. Cox and J. R. Forshaw, Comput. Phys. Commun. 144 (2002) 104 [arXiv:hep-ph/0010303]. 